\title{
Identification of very-low transverse momentum muons in the ATLAS experiment
}

Zhi Zheng*, on behalf of the ATLAS Collaboration

University of Michigan

E-mail: zhengzhi@umich.edu

\begin{abstract}
The standard muon identification in the ATLAS experiment is optimized for muons with transverse momentum $\left(p_{\mathrm{T}}\right)$ above $5 \mathrm{GeV}$. However searches for low mass resonances and many Bphysics studies often need to identify muons with lower $p_{\mathrm{T}}$, where standard algorithms suffer from large backgrounds originating from sources such as hadron decays in flight. A new LowPt selection has been developed to allow optimal muon identification down to $3 \mathrm{GeV}$ in $p_{\mathrm{T}}$ using variables that are sensitive to track kinks in the inner detector and to the imbalance between momentum measured in the inner detector and in the muon spectrometer. The new LowPt identification, analyzed in simulation and in data, shows good efficiencies for muons produced in hard collisions and is effective in rejecting backgrounds.
\end{abstract}

The 39th International Conference on High Energy Physics (ICHEP2018)

4-11 July, 2018

Seoul, Korea

${ }^{*}$ Speaker. 


\section{Introduction}

The identification of low transverse momentum $\left(p_{\mathrm{T}}\right)$ leptons is a crucial aspect of any Bphysics measurement or search for low-mass resonances in the ATLAS experiment[1]. High purity and good momentum resolution can be obtained, with the ATLAS detector, for muon tracks reconstructed in both the inner-detector (ID) and in up to three or four stations of monitored drift tube chambers in the muon spectrometer (MS), allowing for a combined reconstructions (CB)[2]. This is however challenging for $p_{\mathrm{T}}<5 \mathrm{GeV}$ muon tracks because of the sizable amount of energy lost in the calorimeter system before reaching the MS. The LowPt identification working-point (LowPt WP) has been developed in order to obtain optimal muon identification down to a $p_{\mathrm{T}}$ of $3 \mathrm{GeV}$. This was possible thanks to the use of a set of key variables described in Section 2. The details of the selection requirements used for the LowPt WP and its performance in data and simulation are described in Section 3.

\section{Key Variables for Optimal Identification of Low- $p_{\mathbf{T}}$ Muons}

Three variables with good discrimination power between the reconstruction of prompt muon tracks and mis-identified hadron tracks (fakes) have been investigated[3]. Momentum balance significance (MBS) is defined as momentum difference between the ID and MS standalone measurements with respect to the uncertainty sigma on energy lost in the calorimeter system. The scattering neighbor significance (SNS) is a variable estimating the significance of a change in trajectory (kink) along the track, expected in the presence of a hadron decaying to a muon. It is computed considering pairs of adjacent hits along the track, and evaluating the significance of the angular distance in the bending plane between the two half tracks ending/starting at each of them. The SNS is the largest value of such significances over the whole track. The scattering curvature significance (SCS) is computed as the normalized integral of the scattering angle significances, corrected for large kinks along the trajectory. Figure 1 shows the distributions of the described variables for prompt muons and for fakes in simulated $t \bar{t}$ events.


Figure 1: Simulated distributions, in $t \bar{t}$ events, of the MBS (left), SNS (centre) and SCS (right) for tracks associated to prompt muons (blue) and tracks associated to hadron fakes (red).

\section{LowPt Identification Working Point Definition and Performance}

For the LowPt WP, only CB tracks are used. For $|\eta|<1.3$, at least one MS station is required, for $1.3<|\eta|<1.55$, at least two MS stations are required, for $|\eta|>1.55$, the Medium WP[2] 
requirements are applied. Moreover, a very loose selection on variables described in Section 2 is used to suppress fakes: $|\mathrm{MBS}|<3.0,|\mathrm{SNS}|<3.0$ and $|\mathrm{SCS}|<3.0$. The expected probabilities for muons and fakes to satisfy the LowPt WP are shown in Figure 2a for simulated $t \bar{t}$ events as a function of the track $\eta$. Compared with the Medium WP, the LowPt WP recovers efficiency for muon in the barrel region at a cost of moderately increased misidentification probability (fake rate), and suppresses fake rate in end-cap region with a few percent loss of efficiency.

Muon reconstruction efficiencies are measured using a tag-and-probe method [2] for $J / \psi \rightarrow$ $\mu^{+} \mu^{-}$decays. In Figure 2b, results obtained for the LowPt WP are found to be in excellent agreement with the prediction of the detector simulation. The ratio between efficiencies obtained in collision data and simulation is defined as the efficiency scale factor. The available statistics are the dominant source of uncertainties at low $p_{\mathrm{T}}$, and dedicated triggers can be used to reduce the statistical uncertainties.

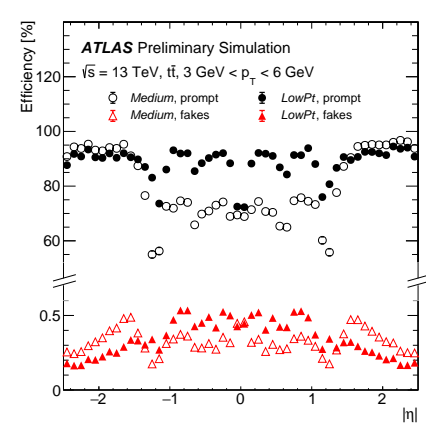

(a) expected efficiency vs. $\eta$

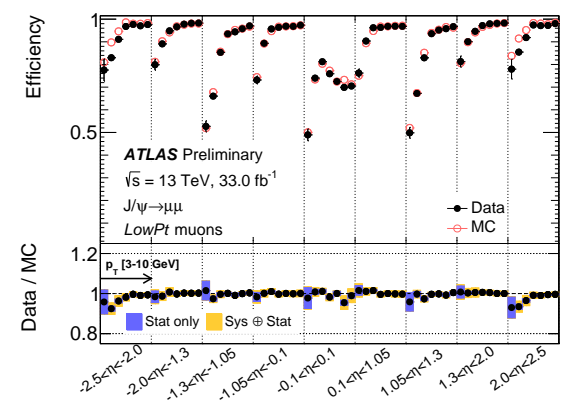

(b) reconstruction efficiency

Figure 2: (a) Expected efficiency as a function of $\eta$ fulfilling the LowPt (filled markers) and Medium (empty markers) requirements in simulated $t \bar{t}$ events. (b) Muon reconstruction efficiencies for the LowPt WP measured using $J / \psi \rightarrow \mu^{+} \mu^{-}$events as a function of the muon $\eta$ and for different $p_{\mathrm{T}}$ ranges, up to $10 \mathrm{GeV}$.

\section{Conclusion}

A new and optimal set of identification criteria, the LowPt WP, has been developed. It allows for a muon selection efficiency of about $90 \%$ for $p_{\mathrm{T}}$ below $5 \mathrm{GeV}$ with an expected hadron misidentification probability below $0.5 \%$. The efficiency measurement has been extended down to $3 \mathrm{GeV}$ with good agreement between data and simulation.

\section{References}

[1] ATLAS Collaboration, The ATLAS Experiment at the CERN Large Hadron Collider, JINST 3 (2008) S08003.

[2] ATLAS Collaboration, "Muon reconstruction performance of the ATLAS detector in proton-proton collision data at $\sqrt{s}=13$ TeV," Eur. Phys. J. C 76, no. 5, 292 (2016)

[3] ATLAS Collaboration, Preliminary Figures from 2016 Collision Data at $13 \mathrm{TeV}$ (2018):

https://twiki.cern.ch/twiki/bin/view/AtlasPublic/MuonPerformancePublicPlots 